\title{
Certain Chebyshev Type Integral Inequalities Involving Hadamard's Fractional Operators
}

\author{
Sotiris K. Ntouyas, ${ }^{1}$ Sunil D. Purohit, ${ }^{2}$ and Jessada Tariboon ${ }^{3}$ \\ ${ }^{1}$ Department of Mathematics, University of Ioannina, 45110 Ioannina, Greece \\ ${ }^{2}$ Department of Basic Sciences (Mathematics), College of Technology and Engineering, M.P. University of Agriculture and Technology, \\ Udaipur 313001, India \\ ${ }^{3}$ Nonlinear Dynamic Analysis Research Center, Department of Mathematics, Faculty of Applied Science, \\ King Mongkut's University of Technology North Bangkok, Bangkok 10800, Thailand
}

Correspondence should be addressed to Jessada Tariboon; jessadat@kmutnb.ac.th

Received 1 April 2014; Accepted 28 April 2014; Published 11 May 2014

Academic Editor: J. C. Prajapati

Copyright (C) 2014 Sotiris K. Ntouyas et al. This is an open access article distributed under the Creative Commons Attribution License, which permits unrestricted use, distribution, and reproduction in any medium, provided the original work is properly cited.

We establish certain new fractional integral inequalities for the differentiable functions whose derivatives belong to the space $L_{p}([1, \infty))$, related to the weighted version of the Chebyshev functional, involving Hadamard's fractional integral operators. As an application, particular results have been also established.

\section{Introduction}

In 1882, Chebyshev [1] gave the following inequality.

If $f, g:[a, b] \rightarrow \mathbb{R}^{+}$are absolutely continuous functions, whose first derivatives $f^{\prime}$ and $g^{\prime}$ are bounded and

$$
\begin{aligned}
T(f, g)= & \frac{1}{b-a} \int_{a}^{b} f(t) g(t) d t \\
& -\left(\frac{1}{b-a} \int_{a}^{b} f(t) d t\right)\left(\frac{1}{b-a} \int_{a}^{b} g(t) d t\right),
\end{aligned}
$$

then

$$
|T(f, g)| \leq \frac{1}{12}(b-a)^{2}\left\|f^{\prime}\right\|_{\infty}\left\|g^{\prime}\right\|_{\infty},
$$

where $\|\cdot\|_{\infty}$ denotes the norm in $L_{\infty}[a, b]$

The Chebyshev functional (1) has many applications in numerical quadrature, transform theory, probability, study of existence for solutions of differential equations, and in statistical problems. Moreover, in the theory of approximations, under various assumptions (Chebyshev inequalities, Grüss inequality, etc.), Chebyshev functionals are useful to give lower bound or upper bounds for the functions.
Therefore, in the literature, we found several extensions and generalizations of these classical integral inequalities, including fractional calculus and $q$-calculus operators also (see [2-14]).

Our work in the present paper is based on a weighted version of the Chebyshev functional (see [1]):

$$
\begin{aligned}
T(f, g, p)= & \int_{a}^{b} p(t) d t \int_{a}^{b} f(t) g(t) p(t) d t \\
& -\int_{a}^{b} f(t) p(t) d t \int_{a}^{b} g(t) p(t) d t,
\end{aligned}
$$

where $f$ and $g$ are two integrable functions on $[a, b]$ and $p(t)$ is a positive and integrable function on $[a, b]$. In 2000, Dragomir [15] derived the following inequality:

$$
\begin{aligned}
& 2|T(f, g, p)| \\
& \quad \leq\left\|f^{\prime}\right\|_{r}\left\|g^{\prime}\right\|_{s}\left[\iint_{a}^{b}|x-y| p(x) p(y) d x d y\right],
\end{aligned}
$$

where $f$ and $g$ are two differentiable functions and $f^{\prime} \in$ $L_{r}(a, b), g^{\prime} \in L_{s}(a, b), r>1$, and $r^{-1}+s^{-1}=1$. Recently, 
Dahmani et al. [16] added one more dimension to this study by introducing generalization of inequality (4), involving Riemann-Liouville fractional integrals. Moreover, Purohit and Raina [17-19] and Baleanu et al. [20, 21] introduced certain generalized integral inequalities for synchronous functions, involving the various fractional hypergeometric integral operators, while Tariboon et al. [22] studied Riemann-Liouville fractional integral inequalities.

In 1892, Hadamard [23] introduced a fractional derivative, which differs from the Riemann-Liouville and Caputo derivatives in the sense that the kernel of the integral contains logarithmic function of arbitrary exponent. For details and fundamental properties of Hadamard fractional derivative and integral can be found in [24-28]. Recently, some results on fractional integral inequalities have been derived by using Hadamard fractional integrals (see [29-32]).

In this paper, we establish certain integral inequalities related to the weighted Chebyshev's functional (3) in the case of differentiable functions whose derivatives belong to the space $L_{p}([1, \infty))$, involving Hadamard fractional integral operators [23]. We also develop some integral inequalities for the fractional integrals by suitably choosing the function $p(t)$, as special cases of our findings.

Firstly, we mention below the basic definitions and notations of some well-known operators of fractional calculus, which shall be used in the sequel.

The Hadamard fractional integral of order $\alpha \in \mathbb{R}^{+}$of a function $f(t)$, for all $t>1$, is defined as [28]

$$
{ }_{H} J^{\alpha}\{f(t)\}=\frac{1}{\Gamma(\alpha)} \int_{1}^{t}\left(\log \frac{t}{\tau}\right)^{\alpha-1} f(\tau) \frac{d \tau}{\tau} .
$$

Further, the Hadamard fractional derivative of order $\alpha \in[n-$ $1, n), n \in \mathbb{Z}^{+}$, of a function $f(t)$ is given by

$$
{ }_{H} D^{\alpha}\{f(t)\}=\frac{1}{\Gamma(n-\alpha)}\left(t \frac{d}{d t}\right)^{n} \int_{1}^{t}\left(\log \frac{t}{\tau}\right)^{n-\alpha-1} f(\tau) \frac{d \tau}{\tau} .
$$

Our results in this paper are based on the following preliminary assertions giving composition formula of Hadamard fractional integral and derivatives with a power function ([4])

$$
\begin{gathered}
{ }_{H} J^{\alpha}(\log t)^{\mu-1}=\frac{\Gamma(\mu)}{\Gamma(\mu+\alpha)}(\log t)^{\mu+\alpha-1}, \\
{ }_{H} D^{\alpha}(\log t)^{\mu-1}=\frac{\Gamma(\mu)}{\Gamma(\mu-\alpha)}(\log t)^{\mu-\alpha-1},
\end{gathered}
$$

where $0<\alpha<1$.

\section{Main Results}

Our results in this section are related to the Chebyshev's functional (3) in the case of differentiable mappings whose derivatives belong to the space $L_{p}([1, \infty))$ and satisfying Holder's inequality. Here, we obtain certain new integral inequalities which give an estimation for the fractional integral of a product in terms of the product of the individual function fractional integrals, involving Hadamard fractional integral operators.
Theorem 1. Let $p$ be a positive function and let $f$ and $g$ be two differentiable functions on $[1, \infty)$. If $f^{\prime} \in L_{r}([1, \infty)), g^{\prime} \in$ $L_{s}([1, \infty)), r>1, r^{-1}+s^{-1}=1$, then, for all $t>1$ and $\alpha>0$,

$$
\begin{aligned}
& \left.2\right|_{H} J^{\alpha}\{p(t)\}_{H} J^{\alpha}\{p(t) f(t) g(t)\}-{ }_{H} J^{\alpha}\{p(t) f(t)\} \\
& \times{ }_{H} J^{\alpha}\{p(t) g(t)\} \mid \\
& \leq \frac{\left\|f^{\prime}\right\|_{r}\left\|g^{\prime}\right\|_{s}}{\Gamma^{2}(\alpha)} \iint_{1}^{t}\left(\log \frac{t}{\tau}\right)^{\alpha-1}\left(\log \frac{t}{\rho}\right)^{\alpha-1} \frac{p(\tau) p(\rho)}{\tau \rho} \\
& \quad \times|\tau-\rho| d \tau d \rho \\
& \leq\left\|f^{\prime}\right\|_{r}\left\|g^{\prime}\right\|_{s} t\left({ }_{H} J^{\alpha}\{p(t)\}\right)^{2} .
\end{aligned}
$$

Proof. We define

$$
\begin{aligned}
\mathscr{H}(\tau, \rho) & =(f(\tau)-f(\rho))(g(\tau)-g(\rho)), \\
F(t, \tau) & =\frac{(\log (t / \tau))^{\alpha-1}}{\tau \Gamma(\alpha)}, \quad \tau \in(1, t), t>1 .
\end{aligned}
$$

We observe that the function $F(t, \tau)$ remains positive, for all $\tau \in(1, t)(t>1)$. Multiplying both sides of (9) by $F(t, \tau) p(\tau)$ and integrating with respect to $\tau$ from 1 to $t$, we get

$$
\begin{aligned}
& \frac{1}{\Gamma(\alpha)} \int_{1}^{t}\left(\log \frac{t}{\tau}\right)^{\alpha-1} \frac{p(\tau)}{\tau} \mathscr{H}(\tau, \rho) d \tau \\
& ={ }_{H} J^{\alpha}\{p(t) f(t) g(t)\}-f(\rho)_{H} J^{\alpha}\{p(t) g(t)\} \\
& \quad-g(\rho)_{H} J^{\alpha}\{p(t) f(t)\}+f(\rho) g(\rho)_{H} J^{\alpha}\{p(t)\} .
\end{aligned}
$$

Next, multiplying both sides of (11) by $F(t, \rho) p(\rho)$ and integrating with respect to $\rho$ from 1 to $t$, we can write

$$
\begin{gathered}
\frac{1}{\Gamma^{2}(\alpha)} \iint_{1}^{t}\left(\log \frac{t}{\tau}\right)^{\alpha-1}\left(\log \frac{t}{\rho}\right)^{\alpha-1} \frac{p(\tau) p(\rho)}{\tau \rho} \mathscr{H}(\tau, \rho) d \tau d \rho \\
=2\left({ }_{H} J^{\alpha}\{p(t)\}_{H} J^{\alpha}\{p(t) f(t) g(t)\}\right. \\
\left.-{ }_{H} J^{\alpha}\{p(t) f(t)\}_{H} J^{\alpha}\{p(t) g(t)\}\right) .
\end{gathered}
$$

In view of (9), we have

$$
\mathscr{H}(\tau, \rho)=\iint_{\tau}^{\rho} f^{\prime}(y) g^{\prime}(z) d y d z
$$

Using the following Hölder's inequality for $r>1$ and $r^{-1}+$ $s^{-1}=1$,

$$
\begin{array}{r}
\left|\iint_{\tau}^{\rho} f(y) g(z) d y d z\right| \\
\leq\left.\left.\left.\left.\left|\iint_{\tau}^{\rho}\right| f(y)\right|^{r} d y d z\right|^{r^{-1}}\left|\iint_{\tau}^{\rho}\right| g(z)\right|^{s} d y d z\right|^{s^{-1}}, \\
\left(r^{-1}+s^{-1}=1\right),
\end{array}
$$


Abstract and Applied Analysis

3

we obtain

$$
|\mathscr{H}(\tau, \rho)| \leq\left.\left.\left.\left.\left|\iint_{\tau}^{\rho}\right| f^{\prime}(y)\right|^{r} d y d z\right|^{r^{-1}}\left|\iint_{\tau}^{\rho}\right| g^{\prime}(z)\right|^{s} d y d z\right|^{s^{-1}} .
$$

Since

$$
\begin{aligned}
& \left.\left.\left|\iint_{\tau}^{\rho}\right| f^{\prime}(y)\right|^{r} d y d z\right|^{r^{-1}}=\left.\left.|\tau-\rho|^{r^{-1}}\left|\int_{\tau}^{\rho}\right| f^{\prime}(y)\right|^{r} d y\right|^{r^{-1}}, \\
& \left.\left.\left|\iint_{\tau}^{\rho}\right| g^{\prime}(z)\right|^{s} d y d z\right|^{s^{-1}}=\left.\left.|\tau-\rho|^{s^{-1}}\left|\int_{\tau}^{\rho}\right| g^{\prime}(z)\right|^{s} d z\right|^{s^{-1}},
\end{aligned}
$$

therefore, inequality (15) reduces to

$$
|\mathscr{H}(\tau, \rho)| \leq\left.\left.\left.\left.|\tau-\rho|\left|\int_{\tau}^{\rho}\right| f^{\prime}(y)\right|^{r} d y\right|^{r^{-1}}\left|\int_{\tau}^{\rho}\right| g^{\prime}(z)\right|^{s} d z\right|^{s^{-1}} .
$$

It follows from (12) that

$$
\begin{array}{r}
\frac{1}{\Gamma^{2}(\alpha)} \iint_{1}^{t}\left(\log \frac{t}{\tau}\right)^{\alpha-1}\left(\log \frac{t}{\rho}\right)^{\alpha-1} \frac{p(\tau) p(\rho)}{\tau \rho}|\mathscr{H}(\tau, \rho)| d \tau d \rho \\
\leq \frac{1}{\Gamma^{2}(\alpha)} \iint_{1}^{t}\left(\log \frac{t}{\tau}\right)^{\alpha-1}\left(\log \frac{t}{\rho}\right)^{\alpha-1} \frac{p(\tau) p(\rho)}{\tau \rho}|\tau-\rho| \\
\times\left.\left.\left.\left.\left|\int_{\tau}^{\rho}\right| f^{\prime}(y)\right|^{r} d y\right|^{r^{-1}}\left|\int_{\tau}^{\rho}\right| g^{\prime}(z)\right|^{s} d z\right|^{s^{-1}} d \tau d \rho .
\end{array}
$$

Applying again Hölder's inequality on the right-hand side of (18), we get

$$
\begin{gathered}
\frac{1}{\Gamma^{2}(\alpha)} \iint_{1}^{t}\left(\log \frac{t}{\tau}\right)^{\alpha-1}\left(\log \frac{t}{\rho}\right)^{\alpha-1} \frac{p(\tau) p(\rho)}{\tau \rho} \\
\quad \times|\mathscr{H}(\tau, \rho)| d \tau d \rho \\
\leq\left[\frac{1}{\Gamma^{r}(\alpha)} \iint_{1}^{t}\left(\log \frac{t}{\tau}\right)^{\alpha-1}\left(\log \frac{t}{\rho}\right)^{\alpha-1} \frac{p(\tau) p(\rho)}{\tau \rho}|\tau-\rho|\right. \\
\left.\times\left.\left|\int_{\tau}^{\rho}\right| f^{\prime}(y)\right|^{r} d y \mid d \tau d \rho\right]^{r^{-1}} \\
\times\left[\frac{1}{\Gamma^{s}(\alpha)} \iint_{1}^{t}\left(\log \frac{t}{\tau}\right)^{\alpha-1}\left(\log \frac{t}{\rho}\right)^{\alpha-1} \frac{p(\tau) p(\rho)}{\tau \rho}\right. \\
\left.\times\left.|\tau-\rho|\left|\int_{\tau}^{\rho}\right| g^{\prime}(z)\right|^{s} d z \mid d \tau d \rho\right]^{s^{-1}} .
\end{gathered}
$$

In view of the fact that

$$
\left.\left|\int_{\tau}^{\rho}\right| f(y)\right|^{p} d y \mid \leq\|f\|_{p}^{p}
$$

we get

$$
\begin{gathered}
\frac{1}{\Gamma^{2}(\alpha)} \iint_{1}^{t}\left(\log \frac{t}{\tau}\right)^{\alpha-1}\left(\log \frac{t}{\rho}\right)^{\alpha-1} \frac{p(\tau) p(\rho)}{\tau \rho}|\mathscr{H}(\tau, \rho)| d \tau d \rho \\
\leq\left[\frac{\left\|f^{\prime}\right\|_{r}^{r}}{\Gamma^{r}(\alpha)} \iint_{1}^{t}\left(\log \frac{t}{\tau}\right)^{\alpha-1}\left(\log \frac{t}{\rho}\right)^{\alpha-1} \frac{p(\tau) p(\rho)}{\tau \rho}\right. \\
\times|\tau-\rho| d \tau d \rho]^{r^{-1}} \\
\times\left[\frac{\left\|g^{\prime}\right\|_{s}^{s}}{\Gamma^{s}(\alpha)} \iint_{1}^{t}\left(\log \frac{t}{\tau}\right)^{\alpha-1}\left(\log \frac{t}{\rho}\right)^{\alpha-1} \frac{p(\tau) p(\rho)}{\tau \rho}\right. \\
\times|\tau-\rho| d \tau d \rho]^{s^{-1}} .
\end{gathered}
$$

From (21), we obtain

$$
\begin{gathered}
\frac{1}{\Gamma^{2}(\alpha)} \iint_{1}^{t}\left(\log \frac{t}{\tau}\right)^{\alpha-1}\left(\log \frac{t}{\rho}\right)^{\alpha-1} \frac{p(\tau) p(\rho)}{\tau \rho}|\mathscr{H}(\tau, \rho)| d \tau d \rho \\
\leq \frac{\left\|f^{\prime}\right\|_{r}\left\|g^{\prime}\right\|_{s}}{\Gamma^{2}(\alpha)}\left[\iint_{1}^{t}\left(\log \frac{t}{\tau}\right)^{\alpha-1}\left(\log \frac{t}{\rho}\right)^{\alpha-1} \frac{p(\tau) p(\rho)}{\tau \rho}\right. \\
\times|\tau-\rho| d \tau d \rho]^{r^{-1}}
\end{gathered}
$$

$$
\times\left[\iint_{1}^{t}\left(\log \frac{t}{\tau}\right)^{\alpha-1}\left(\log \frac{t}{\rho}\right)^{\alpha-1} \frac{p(\tau) p(\rho)}{\tau \rho}|\tau-\rho| d \tau d \rho\right]^{s^{-1}} \text {. }
$$

Using the relation $r^{-1}+s^{-1}=1$, the above inequality yields to

$$
\begin{gathered}
\frac{1}{\Gamma^{2}(\alpha)} \iint_{1}^{t}\left(\log \frac{t}{\tau}\right)^{\alpha-1}\left(\log \frac{t}{\rho}\right)^{\alpha-1} \frac{p(\tau) p(\rho)}{\tau \rho}|\mathscr{H}(\tau, \rho)| d \tau d \rho \\
\leq \frac{\left\|f^{\prime}\right\|_{r}\left\|g^{\prime}\right\|_{s}}{\Gamma^{2}(\alpha)} \iint_{1}^{t}\left(\log \frac{t}{\tau}\right)^{\alpha-1}\left(\log \frac{t}{\rho}\right)^{\alpha-1} \frac{p(\tau) p(\rho)}{\tau \rho} \\
\times|\tau-\rho| d \tau d \rho .
\end{gathered}
$$

On the other hand, (12) gives

$$
\begin{aligned}
& \left.2\right|_{H} J^{\alpha}\{p(t)\}_{H} J^{\alpha}\{p(t) f(t) g(t)\} \\
& -{ }_{H} J^{\alpha}\{p(t) f(t)\}_{H} J^{\alpha}\{p(t) g(t)\} \mid \\
& \leq \frac{1}{\Gamma^{2}(\alpha)} \iint_{1}^{t}\left(\log \frac{t}{\tau}\right)^{\alpha-1}\left(\log \frac{t}{\rho}\right)^{\alpha-1} \frac{p(\tau) p(\rho)}{\tau \rho} \\
& \times|\mathscr{H}(\tau, \rho)| d \tau d \rho .
\end{aligned}
$$


On making use of (23) and (24), the left-hand side of inequality (8) follows very easily.

Now, to prove the right-hand side of inequality (8), we observe that $1 \leq \tau \leq t, 1 \leq \rho \leq t$, and therefore,

$$
0 \leq|\tau-\rho| \leq t
$$

Evidently, from (23), we get

$$
\begin{gathered}
\frac{1}{\Gamma^{2}(\alpha)} \iint_{1}^{t}\left(\log \frac{t}{\tau}\right)^{\alpha-1}\left(\log \frac{t}{\rho}\right)^{\alpha-1} \frac{p(\tau) p(\rho)}{\tau \rho} \\
\times|\mathscr{H}(\tau, \rho)| d \tau d \rho \\
\leq \frac{\left\|f^{\prime}\right\|_{r}\left\|g^{\prime}\right\|_{s} t}{\Gamma^{2}(\alpha)} \iint_{1}^{t}\left(\log \frac{t}{\tau}\right)^{\alpha-1}\left(\log \frac{t}{\rho}\right)^{\alpha-1} \\
\times \frac{p(\tau) p(\rho)}{\tau \rho} d \tau d \rho \\
\left.=\left\|f^{\prime}\right\|_{r}\left\|g^{\prime}\right\|_{s} t{ }_{H} J^{\alpha}\{p(t)\}\right)^{2},
\end{gathered}
$$

which completes the proof of Theorem 1 .

Now, we establish the following integral inequality, which may be regarded as a generalization of Theorem 1 .

Theorem 2. Let $p$ be a positive function and let $f$ and $g$ be two differentiable functions on $[1, \infty)$. If $f^{\prime} \in L_{r}([1, \infty)), g^{\prime} \in$ $L_{s}([1, \infty)), r>1, r^{-1}+s^{-1}=1$, then

$$
\begin{aligned}
& \mid{ }_{H} J^{\alpha}\{p(t)\}_{H} J^{\beta}\{p(t) f(t) g(t)\} \\
& +{ }_{H} J^{\beta}\{p(t)\}_{H} J^{\alpha}\{p(t) f(t) g(t)\} \\
& -{ }_{H} J^{\alpha}\{p(t) f(t)\}_{H} J^{\beta}\{p(t) g(t)\} \\
& -{ }_{H} J^{\beta}\{p(t) f(t)\}_{H} J^{\alpha}\{p(t) g(t)\} \mid \\
& \leq \frac{\left\|f^{\prime}\right\|_{r}\left\|g^{\prime}\right\|_{s} \iint_{\Gamma}^{t}\left(\log \frac{t}{\tau}\right)^{\alpha-1}\left(\log \frac{t}{\rho}\right)^{\beta-1} \frac{p(\tau) p(\rho)}{\tau \rho}}{\leq\left\|f^{\prime}\right\|_{r}\left\|g^{\prime}\right\|_{s} t_{H} J^{\alpha}\left\{p(t){ }_{H} J^{\beta}\{p(t)\},\right.}
\end{aligned}
$$

for all $t>1, \alpha>0$, and $\beta>0$.

Proof. To prove the above theorem, we use inequality (11). Multiplying both sides of (11) by

$$
\frac{(\log (t / \rho))^{\beta-1} p(\rho)}{\rho \Gamma(\beta)}, \quad \rho \in(1, t), t>1,
$$

which remains positive and integrating with respect to $\rho$ from 1 to $t$, we get

$$
\begin{gathered}
\frac{1}{\Gamma(\alpha) \Gamma(\beta)} \iint_{1}^{t}\left(\log \frac{t}{\tau}\right)^{\alpha-1}\left(\log \frac{t}{\rho}\right)^{\beta-1} \frac{p(\tau) p(\rho)}{\tau \rho} \\
\times \mathscr{H}(\tau, \rho) d \tau d \rho \\
={ }_{H} J^{\alpha}\{p(t)\}_{H} J^{\beta}\{p(t) f(t) g(t)\} \\
+{ }_{H} J^{\beta}\{p(t)\}_{H} J^{\alpha}\{p(t) f(t) g(t)\} \\
-{ }_{H} J^{\alpha}\{p(t) f(t)\}_{H} J^{\beta}\{p(t) g(t)\} \\
-{ }_{H} J^{\beta}\{p(t) f(t)\}_{H} J^{\alpha}\{p(t) g(t)\} .
\end{gathered}
$$

Now making use of (17), (29) gives

$$
\begin{aligned}
\frac{1}{\Gamma(\alpha) \Gamma(\beta)} \iint_{1}^{t}( & \left.\log \frac{t}{\tau}\right)^{\alpha-1}\left(\log \frac{t}{\rho}\right)^{\beta-1} \frac{p(\tau) p(\rho)}{\tau \rho} \\
& \times|\mathscr{H}(\tau, \rho)| d \tau d \rho \\
\leq \frac{1}{\Gamma(\alpha) \Gamma(\beta)} \iint_{1}^{t}( & \left.\log \frac{t}{\tau}\right)^{\alpha-1}\left(\log \frac{t}{\rho}\right)^{\beta-1} \frac{p(\tau) p(\rho)}{\tau \rho} \\
& \times\left.\left.|\tau-\rho|\left|\int_{\tau}^{\rho}\right| f^{\prime}(y)\right|^{r} d y\right|^{r^{-1}} \\
& \times\left.\left.\left|\int_{\tau}^{\rho}\right| g^{\prime}(z)\right|^{s} d z\right|^{s^{-1}} d \tau d \rho .
\end{aligned}
$$

Applying Hölder's inequality on the right-hand side of (30), we get

$$
\begin{aligned}
& \frac{1}{\Gamma(\alpha) \Gamma(\beta)} \iint_{1}^{t}\left(\log \frac{t}{\tau}\right)^{\alpha-1}\left(\log \frac{t}{\rho}\right)^{\beta-1} \frac{p(\tau) p(\rho)}{\tau \rho} \\
& \times|\mathscr{H}(\tau, \rho)| d \tau d \rho \\
& \leq\left[\frac{1}{\Gamma^{r}(\alpha)} \iint_{1}^{t}\left(\log \frac{t}{\tau}\right)^{\alpha-1}\left(\log \frac{t}{\rho}\right)^{\beta-1} \frac{p(\tau) p(\rho)}{\tau \rho}|\tau-\rho|\right. \\
& \left.\times\left.\left|\int_{\tau}^{\rho}\right| f^{\prime}(y)\right|^{r} d y \mid d \tau d \rho\right]^{r^{-1}} \\
& \times\left[\frac{1}{\Gamma^{s}(\beta)} \iint_{1}^{t}\left(\log \frac{t}{\tau}\right)^{\alpha-1}\left(\log \frac{t}{\rho}\right)^{\beta-1} \frac{p(\tau) p(\rho)}{\tau \rho}|\tau-\rho|\right. \\
& \left.\times\left.\left|\int_{\tau}^{\rho}\right| g^{\prime}(z)\right|^{s} d z \mid d \tau d \rho\right]^{s^{-1}}
\end{aligned}
$$


or

$$
\begin{gathered}
\frac{1}{\Gamma(\alpha) \Gamma(\beta)} \iint_{1}^{t}\left(\log \frac{t}{\tau}\right)^{\alpha-1}\left(\log \frac{t}{\rho}\right)^{\beta-1} \frac{p(\tau) p(\rho)}{\tau \rho} \\
\times|\mathscr{H}(\tau, \rho)| d \tau d \rho \\
\leq \frac{\left\|f^{\prime}\right\| r\left\|g^{\prime}\right\|_{s}}{\Gamma(\alpha) \Gamma(\beta)} \iint_{1}^{t}\left(\log \frac{t}{\tau}\right)^{\alpha-1}\left(\log \frac{t}{\rho}\right)^{\beta-1} \frac{p(\tau) p(\rho)}{\tau \rho} \\
\times|\tau-\rho| d \tau d \rho .
\end{gathered}
$$

In view of (29) and (32) and the properties of modulus, one can easily arrive at the left-sided inequality of Theorem 2 . Moreover, we have $1 \leq \tau \leq t, 1 \leq \rho \leq t$; hence,

$$
0 \leq|\tau-\rho| \leq t
$$

Therefore, from (32), we get

$$
\begin{aligned}
& \frac{1}{\Gamma(\alpha) \Gamma(\beta)} \iint_{1}^{t}\left(\log \frac{t}{\tau}\right)^{\alpha-1}\left(\log \frac{t}{\rho}\right)^{\beta-1} \frac{p(\tau) p(\rho)}{\tau \rho} \\
& \quad \times|\mathscr{H}(\tau, \rho)| d \tau d \rho \\
& \leq \frac{\left\|f^{\prime}\right\|\left\|g_{r}^{\prime}\right\|_{s}}{\Gamma(\alpha) \Gamma(\beta)} \iint_{1}^{t}\left(\log \frac{t}{\tau}\right)^{\alpha-1} \times\left(\log \frac{t}{\rho}\right)^{\beta-1} \frac{p(\tau) p(\rho)}{\tau \rho} \\
& \times|\tau-\rho| d \tau d \rho \\
& \leq\left\|f^{\prime}\right\|_{r}\left\|g^{\prime}\right\|_{s} t_{H} J^{\alpha}\{p(t)\}_{H} J^{\beta}\{p(t)\},
\end{aligned}
$$

which completes the proof of Theorem 2.

Remark 3. For $\beta=\alpha$, Theorem 2 immediately reduces to Theorem 1.

\section{Special Cases}

As implications of our main results, we consider some consequent results of Theorems 1 and 2 by suitably choosing the function $p(t)$. Other classes of no weighted inequalities are also obtained. To this end, let us set $p(t)=(\log t)^{\lambda}(\lambda \in$ $[0, \infty), t \in(1, \infty))$; then Theorems 1 and 2 yield the following results.

Corollary 4. Let $f$ and $g$ be two differentiable functions on $[1, \infty)$. If $f^{\prime} \in L_{r}([1, \infty)), g^{\prime} \in L_{s}([1, \infty)), r>1, r^{-1}+s^{-1}=$ 1 , then, for all $t>1, \lambda \in[0, \infty)$ and $\alpha>0$,

$$
\begin{gathered}
2 \mid \frac{\Gamma(1+\lambda)}{\Gamma(1+\lambda+\alpha)}(\log t)^{\lambda+\alpha}{ }_{H} J^{\alpha}\left\{(\log t)^{\lambda} f(t) g(t)\right\} \\
-{ }_{H} J^{\alpha}\left\{(\log t)^{\lambda} f(t)\right\}{ }_{H} J^{\alpha}\left\{(\log t)^{\lambda} g(t)\right\} \mid
\end{gathered}
$$

$$
\begin{gathered}
\leq \frac{\left\|f^{\prime}\right\|_{r}\left\|g^{\prime}\right\|_{s}}{\Gamma^{2}(\alpha)} \iint_{1}^{t}\left(\log \frac{t}{\tau}\right)^{\alpha-1}\left(\log \frac{t}{\rho}\right)^{\alpha-1} \frac{(\log \tau)^{\lambda}(\log \rho)^{\lambda}}{\tau \rho} \\
\times|\tau-\rho| d \tau d \rho \\
\leq\left\|f^{\prime}\right\|_{r}\left\|g^{\prime}\right\|_{s} t \frac{\Gamma^{2}(1+\lambda)}{\Gamma^{2}(1+\lambda+\alpha)}(\log t)^{2 \lambda+2 \alpha} .
\end{gathered}
$$

Corollary 5. Let $f$ and $g$ be two differentiable functions on $[1, \infty)$. If $f^{\prime} \in L_{r}([1, \infty)), g^{\prime} \in L_{s}([1, \infty)), r>1, r^{-1}+s^{-1}=$ 1 , then

$$
\begin{aligned}
& \mid \frac{\Gamma(1+\lambda)}{\Gamma(1+\lambda+\alpha)}(\log t)^{\lambda+\alpha} J_{H}^{\beta}\left\{(\log t)^{\lambda} f(t) g(t)\right\} \\
& +\frac{\Gamma(1+\lambda)}{\Gamma(1+\lambda+\beta)}(\log t)^{\lambda+\beta} J^{\alpha}\left\{(\log t)^{\lambda} f(t) g(t)\right\} \\
& -{ }_{H} J^{\alpha}\left\{(\log t)^{\lambda} f(t)\right\}{ }_{H} J^{\beta}\left\{(\log t)^{\lambda} g(t)\right\} \\
& -{ }_{H} J^{\beta}\left\{(\log t)^{\lambda} f(t){ }_{H} J^{\alpha}\left\{(\log t)^{\lambda} g(t)\right\} \mid\right. \\
& \leq \frac{\left\|f^{\prime}\right\|_{r}\left\|g^{\prime}\right\|_{s} \iint_{t}^{t}\left(\log \frac{t}{\tau}\right)^{\alpha-1}\left(\log \frac{t}{\rho}\right)^{\beta-1} \frac{(\log \tau)^{\lambda}(\log \rho)^{\lambda}}{\tau(\beta)} \int_{1}}{\times|\tau-\rho| d \tau d \rho} \\
& \leq\left\|f^{\prime}\right\|_{r}\left\|g^{\prime}\right\|_{s} t \frac{\Gamma^{2}(1+\lambda)}{\Gamma(1+\lambda+\alpha) \Gamma(1+\lambda+\beta)}(\log t)^{2 \lambda+\alpha+\beta},
\end{aligned}
$$

for all $t>1, \alpha>0, \beta>0$, and $\lambda \in[0, \infty)$.

Further, if we put $\lambda=0$ in Corollaries 4 and 5 (or set $p(t)=1$ in Theorems 1 and 2 ), we obtain the following results.

Corollary 6. Let $f$ and $g$ be two differentiable functions on $[1, \infty)$. If $f^{\prime} \in L_{r}([1, \infty)), g^{\prime} \in L_{s}([1, \infty)), r>1, r^{-1}+s^{-1}=$ 1 , then, for all $t>1$ and $\alpha>0$,

$$
\begin{aligned}
& 2\left|\frac{(\log t)^{\alpha}}{\Gamma(1+\alpha)} H^{\alpha}\{f(t) g(t)\}-{ }_{H} J^{\alpha}\{f(t)\}_{H} J^{\alpha}\{g(t)\}\right| \\
& \quad \leq \frac{\left\|f^{\prime}\right\|_{r}\left\|g^{\prime}\right\|_{s}}{\Gamma^{2}(\alpha)} \int_{1}^{t}\left(\log \frac{t}{\tau}\right)^{\alpha-1}\left(\log \frac{t}{\rho}\right)^{\alpha-1} \frac{|\tau-\rho|}{\tau \rho} d \tau d \rho \\
& \quad \leq\left\|f^{\prime}\right\|_{r}\left\|g^{\prime}\right\|_{s} \frac{t(\log t)^{2 \alpha}}{\Gamma^{2}(1+\alpha)}
\end{aligned}
$$

Corollary 7. Let $f$ and $g$ be two differentiable functions on $[1, \infty)$. If $f^{\prime} \in L_{r}([1, \infty)), g^{\prime} \in L_{s}([1, \infty)), r>1, r^{-1}+s^{-1}=$ 1 , then

$$
\begin{aligned}
& \mid \frac{(\log t)^{\alpha}}{\Gamma(1+\alpha)} J^{\beta}\{f(t) g(t)\}+\frac{(\log t)^{\beta}}{\Gamma(1+\beta)} H^{\alpha}\{f(t) g(t)\} \\
& \quad-{ }_{H} J^{\alpha}\{f(t)\}_{H} J^{\beta}\{g(t)\}-{ }_{H} J^{\beta}\{f(t)\}_{H} J^{\alpha}\{g(t)\} \mid
\end{aligned}
$$




$$
\begin{aligned}
& \leq \frac{\left\|f^{\prime}\right\|_{r}\left\|g^{\prime}\right\|_{s}}{\Gamma(\alpha) \Gamma(\beta)} \iint_{1}^{t}\left(\log \frac{t}{\tau}\right)^{\alpha-1}\left(\log \frac{t}{\rho}\right)^{\beta-1} \frac{|\tau-\rho|}{\tau \rho} d \tau d \rho \\
& \leq\left\|f^{\prime}\right\|_{r}\left\|g^{\prime}\right\|_{s} \frac{t(\log t)^{\alpha+\beta}}{\Gamma(1+\alpha) \Gamma(1+\beta)},
\end{aligned}
$$

for all $t>1, \alpha>0$, and $\beta>0$.

We conclude our paper by remarking that we have introduced new general Chebyshev type inequalities involving Hadamard fractional integral operators. By suitably specializing the arbitrary function $p(t)$, one can further easily obtain additional fractional integral inequalities from our main results (Theorems 1 and 2).

\section{Conflict of Interests}

The authors declare that there is no conflict of interests regarding the publication of this paper.

\section{Acknowledgments}

The research of J. Tariboon is supported by King Mongkut's University of Technology North Bangkok, Thailand. Sotiris K. Ntouyas is a member of Nonlinear Analysis and Applied Mathematics- (NAAM-) Research Group at King Abdulaziz University, Jeddah, Saudi Arabia.

\section{References}

[1] P. L. Chebyshev, "Sur les expressions approximatives des integrales definies par les autres prises entre les mêmes limites," Proceedings of the American Mathematical Society, vol. 2, pp. 9398, 1882.

[2] P. Cerone and S. S. Dragomir, "New upper and lower bounds for the Chebyshev functional," Journal of Inequalities in Pure and Applied Mathematics, vol. 3, article 77, 2002.

[3] D. S. Mitrinović, J. E. Pečarić, and A. M. Fink, Classical and New Inequalities in Analysis, vol. 61, Kluwer Academic, Dordrecht, The Netherlands, 1993.

[4] G. A. Anastassiou, Fractional Differentiation Inequalities, Springer, Dordrecht, The Netherlands, 2009.

[5] G. A. Anastassiou, Advances on Fractional Inequalities, Briefs in Mathematics, Springer, New York, NY, USA, 2011.

[6] J. Pečarić and I. Perić, "Identities for the Chebyshev functional involving derivatives of arbitrary order and applications," Journal of Mathematical Analysis and Applications, vol. 313, no. 2, pp. 475-483, 2006.

[7] S. Belarbi and Z. Dahmani, "On some new fractional integral inequalities," Journal of Inequalities in Pure and Applied Mathematics, vol. 10, no. 3, article 86, 2009.

[8] Z. Dahmani and L. Tabharit, "Certain inequalities involving fractional integrals," Journal of Advanced Research in Scientific Computing, vol. 2, no. 1, pp. 55-60, 2010.

[9] S. L. Kalla and A. Rao, "On Grüss type inequality for a hypergeometric fractional integral," Le Matematiche, vol. 66, no. 1, pp. 57-64, 2011.
[10] H. Öğünmez and U. M. Özkan, "Fractional quantum integral inequalities," Journal of Inequalities and Applications, vol. 2011, Article ID 787939, 7 pages, 2011.

[11] W. T. Sulaiman, "Some new fractional integral inequalities," Journal of Mathematical Analysis, vol. 2, no. 4, pp. 23-28, 2011.

[12] M. Z. Sarikaya and H. Ogunmez, "On new inequalities via Riemann-Liouville fractional integration," Abstract and Applied Analysis, vol. 2012, Article ID 428983, 10 pages, 2012.

[13] M. Z. Sarikaya, E. Set, H. Yaldiz, and N. Basak, "HermiteHadamards inequalities for fractional integrals and related fractional inequalities," Mathematical and Computer Modelling, vol. 57, no. 9-10, pp. 2403-2407, 2013.

[14] Z. Dahmani and A. Benzidane, "On a class of fractional qintegral inequalities," Malaya Journal of Matematik, vol. 3, no. 1, pp. 1-6, 2013.

[15] S. S. Dragomir, "Some integral inequalities of Grüss type," Indian Journal of Pure and Applied Mathematics, vol. 31, no. 4, pp. 397-415, 2000.

[16] Z. Dahmani, O. Mechouar, and S. Brahami, "Certain inequalities related to the Chebyshev's functional involving a RiemannLiouville operator," Bulletin of Mathematical Analysis and Applications, vol. 3, no. 4, pp. 38-44, 2011.

[17] S. D. Purohit and R. K. Raina, "Chebyshev type inequalities for the Saigo fractional integrals and their q-analogues," Journal of Mathematical Inequalities, vol. 7, no. 2, pp. 239-249, 2013.

[18] S. D. Purohit, F. Uçar, and R. K. Yadav, "On fractional integral inequalities and their q-analogues," Revista Tecnocientifica URU. In press.

[19] S. D. Purohit and R. K. Raina, "Certain fractional integral inequalities involving the Gauss hypergeometric function," Revista Técnica de la Facultad de Ingeniería Universidad del Zulia. In press.

[20] D. Baleanu and S. D. Purohit, "Chebyshev type integral inequalities involving the fractional hypergeometric operators," Abstract and Applied Analysis, vol. 2014, Article ID 609160, 10 pages, 2014.

[21] D. Baleanu, S. D. Purohit, and P. Agarwal, "On fractional integral inequalities involving hypergeometric operators," Chinese Journal of Mathematics, vol. 2014, Article ID 609476, 5 pages, 2014.

[22] J. Tariboon, S. K. Ntouyas, and W. Sudsutad, "Some new Riemann-Liouville fractional integral inequalities," International Journal of Mathematics and Mathematical Sciences, vol. 2014, Article ID 869434, 6 pages, 2014.

[23] J. Hadamard, "Essai sur l'etude des fonctions donnees par leur developpment de Taylor," Journal de Mathématiques Pures et Appliquées, vol. 8, pp. 101-186, 1892.

[24] A. A. Kilbas, "Hadamard-type fractional calculus," Journal of the Korean Mathematical Society, vol. 38, no. 6, pp. 1191-1204, 2001.

[25] P. L. Butzer, A. A. Kilbas, and J. J. Trujillo, "Compositions of Hadamard-type fractional integration operators and the semigroup property," Journal of Mathematical Analysis and Applications, vol. 269, no. 2, pp. 387-400, 2002.

[26] P. L. Butzer, A. A. Kilbas, and J. J. Trujillo, "Fractional calculus in the Mellin setting and Hadamard-type fractional integrals," Journal of Mathematical Analysis and Applications, vol. 269, no. 1, pp. 1-27, 2002.

[27] P. L. Butzer, A. A. Kilbas, and J. J. Trujillo, "Mellin transform analysis and integration by parts for Hadamard-type fractional integrals," Journal of Mathematical Analysis and Applications, vol. 270, no. 1, pp. 1-15, 2002. 
[28] A. A. Kilbas, H. M. Srivastava, and J. J. Trujillo, Theory and Applications of Fractional Differential Equations, vol. 204 of North-Holland Mathematics Studies, Elsevier, Amsterdam, The Netherlands, 2006.

[29] V. L. Chinchane and D. B. Pachpatte, "A note on some integral inequalities via Hadamard integral," Journal of Fractional Calculus and Applications, vol. 4, pp. 1-5, 2013.

[30] V. L. Chinchane and D. B. Pachpatte, "On some integral inequalities using Hadamard fractional integral," Malaya Journal of Matematik, vol. 1, pp. 62-66, 2012.

[31] B. Sroysang, "A study on Hadamard fractional integral," International Journal of Mathematical Analysis, vol. 7, no. 37-40, pp. 1903-1906, 2013.

[32] W. Sudsutad, S. K. Ntouyas, and J. Tariboon, "Fractional integral inequalities via Hadamard's fractional integral," Abstract and Applied Analysis, vol. 2014, Article ID 563096, 11 pages, 2014. 


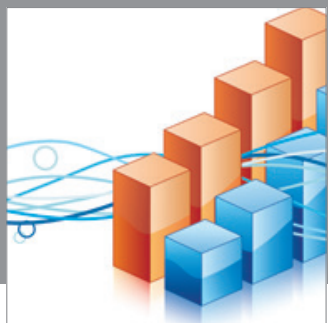

Advances in

Operations Research

mansans

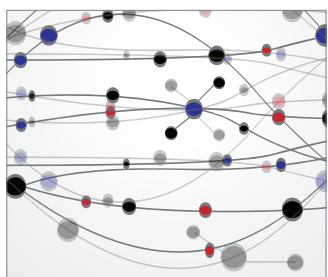

The Scientific World Journal
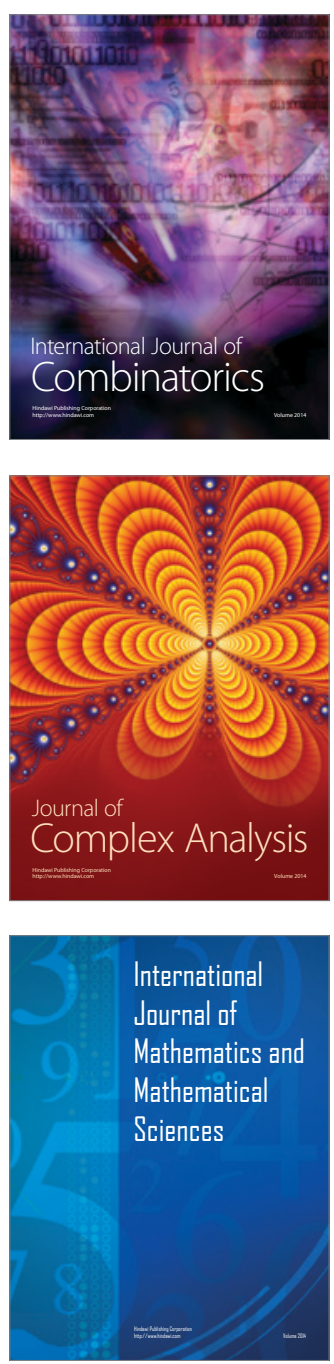
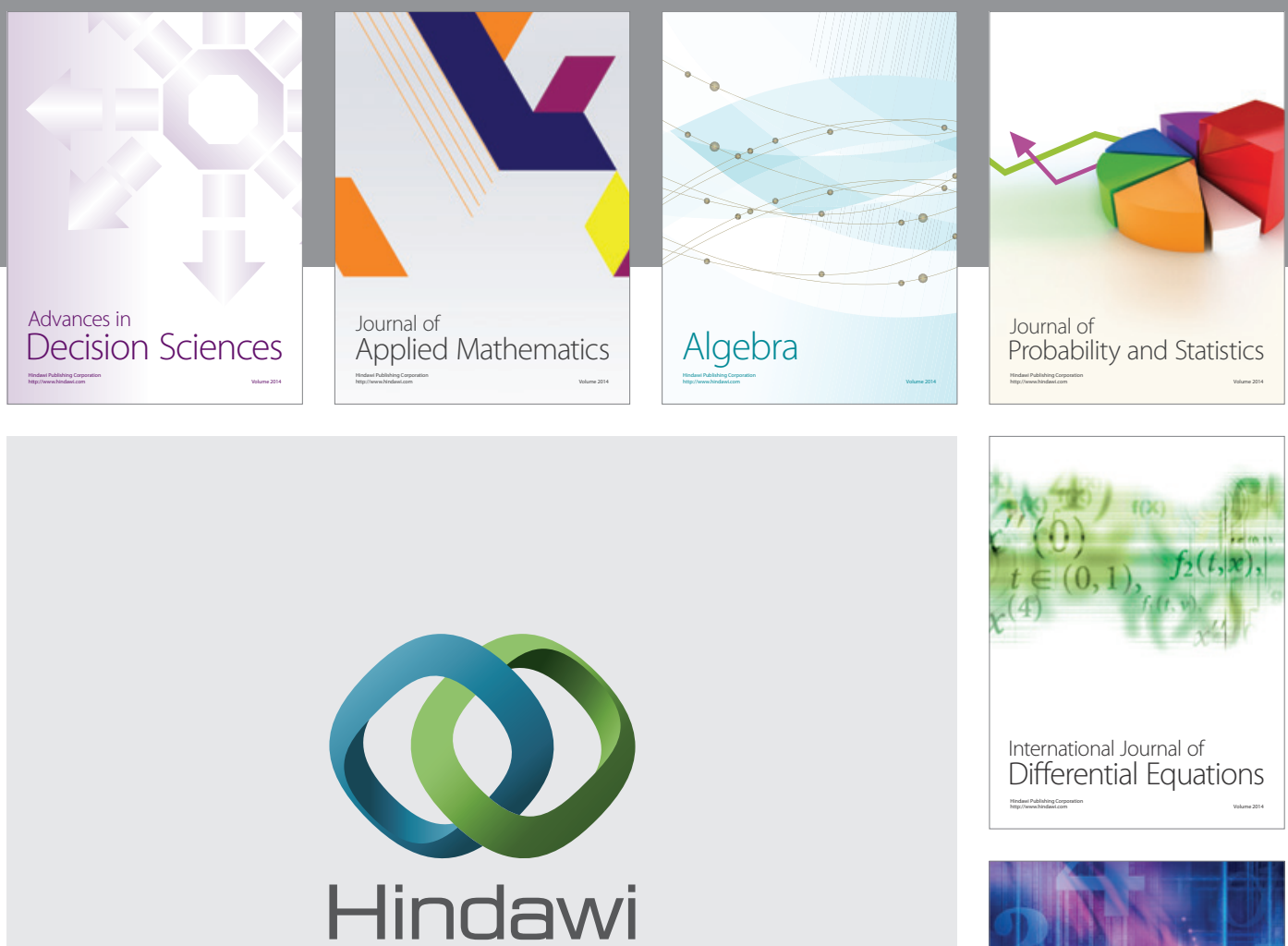

Submit your manuscripts at http://www.hindawi.com
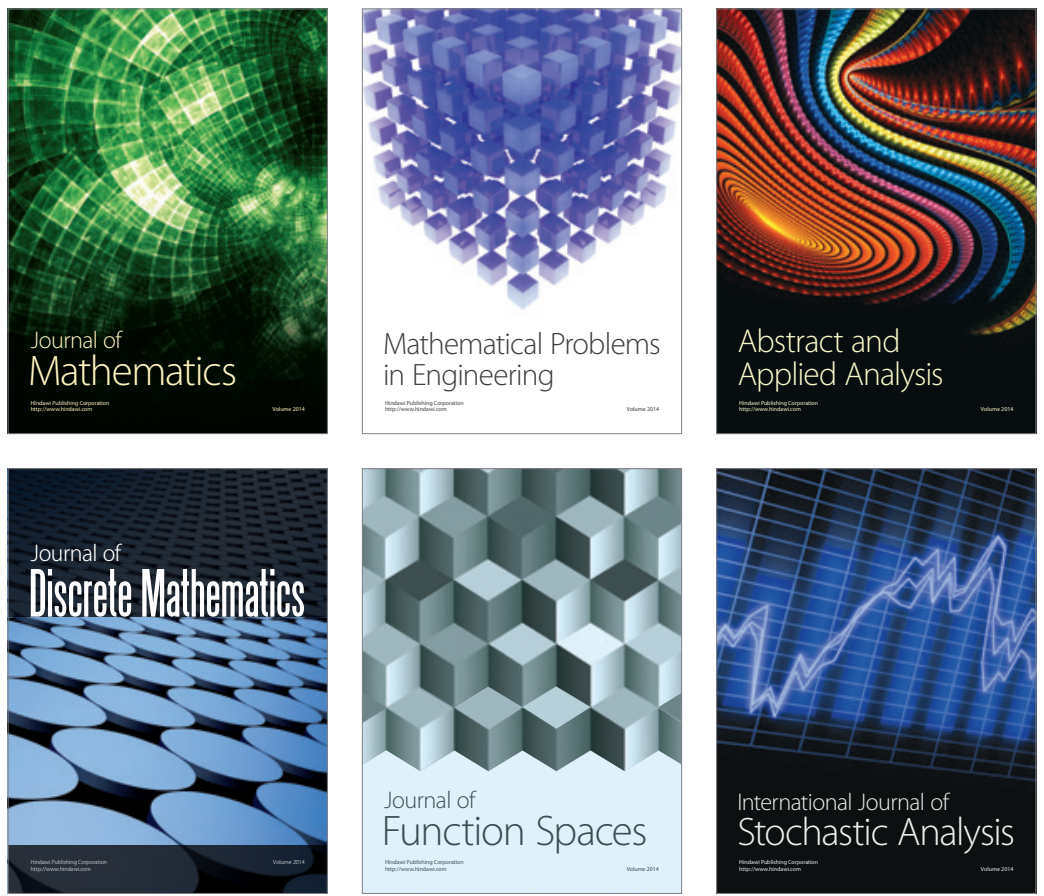

Journal of

Function Spaces

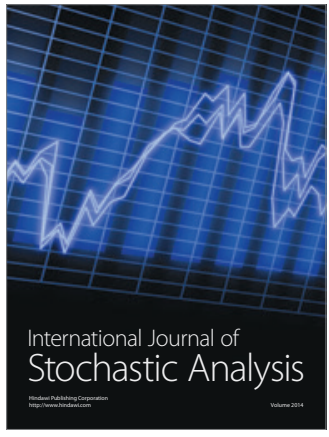

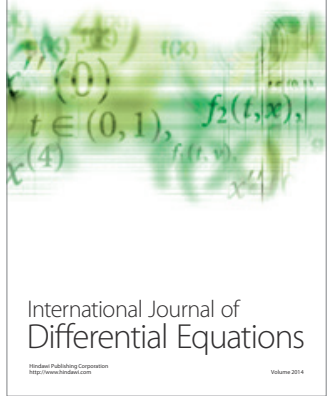
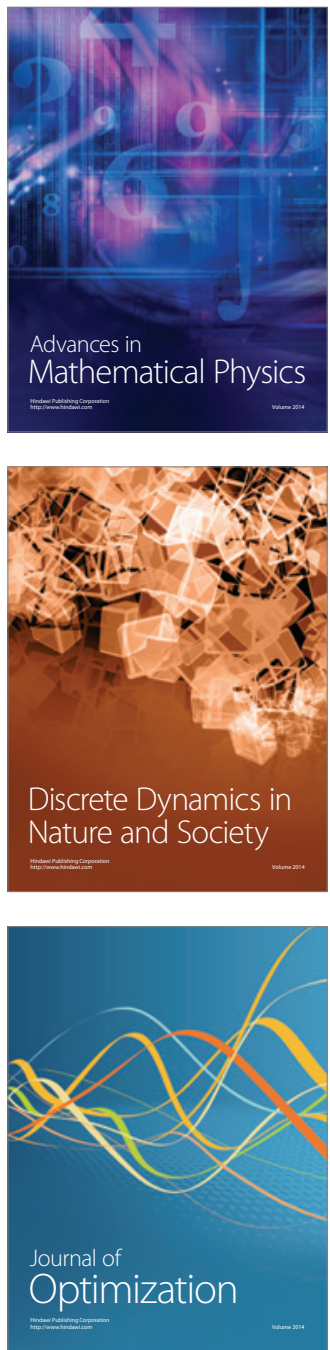